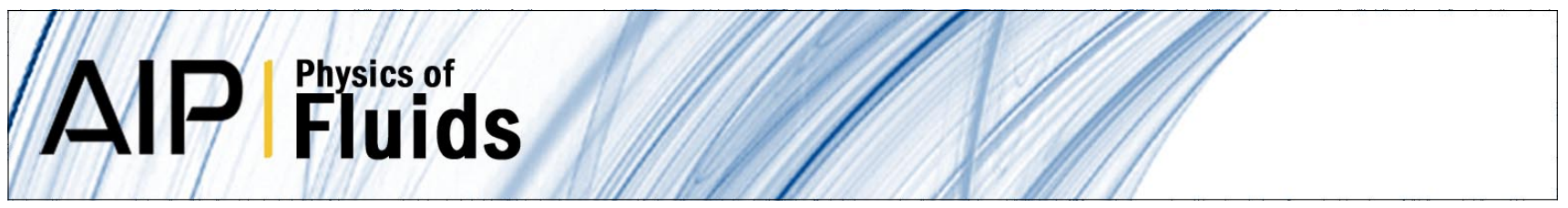

\title{
Dynamics of freely moving plates connected by a shallow liquid bridge
}

Amir Gat, Homayun Navaz, and Morteza Gharib

Citation: Phys. Fluids 23, 097101 (2011); doi: 10.1063/1.3643289

View online: http://dx.doi.org/10.1063/1.3643289

View Table of Contents: http://pof.aip.org/resource/1/PHFLE6/v23/i9

Published by the American Institute of Physics.

\section{Related Articles}

Adaptive beam tracking and steering via electrowetting-controlled liquid prism Appl. Phys. Lett. 99, 191108 (2011)

A study on the dynamic behaviors of water droplets impacting nanostructured surfaces

AlP Advances 1, 042139 (2011)

Hydrophobic interactions in presence of osmolytes urea and trimethylamine- $\mathrm{N}$-oxide

J. Chem. Phys. 135, 174501 (2011)

Wetting on smooth micropatterned defects

Appl. Phys. Lett. 99, 184101 (2011)

Highly effective gold nanoparticle-enhanced biosensor array on the wettability controlled substrate by wiping J. Appl. Phys. 110, 084701 (2011)

\section{Additional information on Phys. Fluids}

Journal Homepage: http://pof.aip.org/

Journal Information: http://pof.aip.org/about/about_the_journal

Top downloads: http://pof.aip.org/features/most_downloaded

Information for Authors: http://pof.aip.org/authors

\section{ADVERTISEMENT}

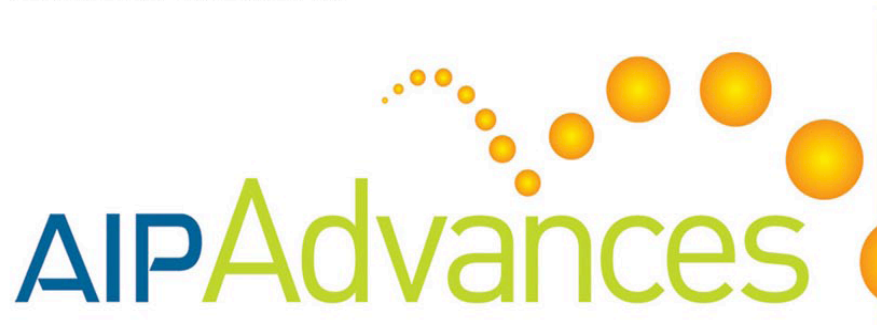

Submit Now

Explore AIP's new

open-access journal

Article-level metrics now available

Join the conversation!

Rate \& comment on articles 


\title{
Dynamics of freely moving plates connected by a shallow liquid bridge
}

\author{
Amir Gat, ${ }^{1}$ Homayun Navaz, ${ }^{2}$ and Morteza Gharib ${ }^{1}$ \\ ${ }^{1}$ Graduate Aeronautical Laboratories, California Institute of Technology, Pasadena, California 91125, USA \\ ${ }^{2}$ Department of Mechanical Engineering, Kettering University, Flint, Michigan 48504, USA
}

(Received 14 March 2011; accepted 1 September 2011; published online 30 September 2011)

\begin{abstract}
We study the dynamics of freely moving plates connected by a shallow liquid bridge via analytic and experimental methods. The gap between the plates is used as a small parameter within a lubrication approximation, reducing the problem to an Abel equation of the second kind. Analysis of the governing differential equation yields two novel physical phenomena: (1) An impulse-like peak in the force applied by the liquid bridge on the plates, obtained from a uniform asymptotic solution for small capillary numbers. (2) Both linear and non-linear oscillations of the system for the case of surfaces with low wettability, obtained from small perturbations of the system around the equilibrium point. An experimental setup examining the motion of freely moving plates was constructed, yielding experimental data which compared favorably with the analytic results and specifically displayed the predicted oscillations and impulse-like peak of the applied force. The application of the current analysis to the manipulation of solid bodies and possible future research directions are discussed. (C) 2011 American Institute of Physics. [doi:10.1063/1.3643289]
\end{abstract}

\section{INTRODUCTION}

We study the dynamics of freely moving solid plates connected by a shallow liquid bridge. This problem is relevant to the adhesion ${ }^{1}$ and to the manipulation of solid bodies by capillary forces (specifically to the release scheme of the manipulated particles ${ }^{2-5}$ ), flip-chip applications ${ }^{6}$ and to spreading of contamination on a freely moving solid in contact with a flat plane filled with contaminated droplets. The dynamics of the solid bodies affects the droplet topology and will thus change the surface area in contact with the droplet and the pressure distribution within the liquid, thus affecting the spreading of contamination. ${ }^{7}$

The forces applied by liquid bridges connected to static supporting surfaces were studied extensively. ${ }^{8}$ However, in many applications it is common that at least one of the bodies is moving, and thus, the motion of the solid body may be influenced by the forces associated with the liquid bridge. $^{9-11}$ Pitois et al. studied $^{12}$ the forces applied by a liquid bridge connecting two spheres moving at a constant speed relative to each other. Similarly, Meurisse and Querry studied ${ }^{13}$ the effects of liquid bridges connecting two parallel flat plates, moving perpendicularly to the plane of the plates, at a constant speed or at a constant force applied on the liquid. Both Pitois et al. and Meurisse and Querry observed a rapid change from attractive force due to capillary effects to repulsive force due to viscous effects for the case of surfaces approaching each other at a constant speed. De Souza et al. studied $^{14}$ the effect of contact angle hysteresis on the capillary forces in the absence of significant viscous forces and obtained good agreement between the experimental data and the existing models. Capillary force measurement of liquid bridges was examined and shown to be a reliable method for estimating advancing and receding wetting angles.

In this work we relate the effects of the momentum of the solid plates to the flow field within the liquid bridge. The inclusion of the solid plate momentum changes the order of the governing ordinary differential equation, relative to existing works. ${ }^{12,13}$ Hence, the obtained model is not only more accurate, but fundamentally different and reveals novel phenomena that could not be described without the inclusion of momentum effects, such as impulse like peak in the force applied by the liquid bridge and linear and non-linear oscillations of the system.

\section{ANALYSIS}

\section{A. Problem definition}

The motion of an incompressible Newtonian fluid is governed by the Navier-Stokes equations, consisting of conservation of mass,

$$
\tilde{\nabla} \cdot \tilde{\mathbf{u}}=0
$$

and conservation of momentum,

$$
\rho \frac{D \tilde{\mathbf{u}}}{D t}=-\tilde{\nabla} \tilde{p}+\mu \tilde{\nabla}^{2} \tilde{\mathbf{u}}+\tilde{\mathbf{B}}
$$

where $\rho$ is density, $p$ is pressure, $t$ is time, $\mathbf{u}$ is the velocity, $\mathbf{B}$ is body forces, and $\mu$ is viscosity (tildes denote dimensional variables). The motion of the center of mass of a rigid solid body, in contact with the surrounding liquid and gas, is governed by

$$
\frac{\partial}{\partial t}\left(\tilde{M} \tilde{\mathbf{u}}_{c}\right)=\oiiint_{S}\left(\tilde{p} \cdot \hat{\mathbf{n}}_{s}\right) d A+\widetilde{\mathbf{F}}
$$

where $M$ is the mass of the body, $\mathbf{u}_{\mathbf{c}}$ is the velocity of the center of mass, $\hat{\mathbf{n}}_{s}$ is a unit vector perpendicular and pointing inward to the surface of the solid body and $\mathbf{F}$ is the sum of the external and body forces.

We limit our analysis to the case of a shallow liquid bridge connected with two solid plates. External forces $F_{1}$ 
and $F_{2}$ are applied to the center of mass of the solid bodies in the $z$ direction (geometry and coordinates are illustrated in Fig. 1) and the problem is assumed axi-symmetric with regard to the center of the liquid bridge. The relevant boundary conditions supplementing the governing equations are no-penetration,

$$
\tilde{v}\left(\tilde{z}=\tilde{s}_{1}\right)=\frac{\partial \tilde{s}_{1}}{\partial \tilde{t}}, \quad \tilde{v}\left(\tilde{z}=\tilde{s}_{2}\right)=\frac{\partial \tilde{s}_{2}}{\partial \tilde{t}},
$$

no-slip at the solid boundaries,

$$
\tilde{u}\left(\tilde{z}=\tilde{s}_{1}\right)=0, \quad \tilde{u}\left(\tilde{z}=\tilde{s}_{2}\right)=0,
$$

the dynamic stress balance at the free surface $r=r_{d}(z, t)$ (Ref. 15),

$$
\left(\tilde{\tau}^{*}-\mathbf{I} \tilde{p}^{*}\right) \cdot \hat{\mathbf{n}}-(\tilde{\tau}-\mathbf{I} \tilde{p}) \cdot \hat{\mathbf{n}}+\gamma \tilde{k} \hat{\mathbf{n}}=0 \text { at } r=r_{d}(z, t),
$$

the kinematic boundary liquid-gas condition at the free surface $\tilde{\mathbf{u}}\left(r_{d}\right)=\tilde{\mathbf{u}}^{*}\left(r_{d}\right)$, the kinematic condition for the position of the free surface, ${ }^{16}$

$$
\frac{1}{\left|\Delta\left(r-r_{d}(z, t)\right)\right|} \frac{\partial\left(r-r_{d}(z, t)\right)}{\partial t}+\mathbf{u} \cdot \mathbf{n}=0 \text { at } r=r_{d}(z, t),
$$

and initial conditions for the positions $s_{1}(t=0), s_{2}(t=0)$ and speeds $\partial s_{1}(t=0) / \partial t, \partial s_{2}(t=0) / \partial t$ of the solid bodies, where the superscript $*$ denotes the properties of the surrounding gas, $u$ is the radial speed, $v$ is the axial speed, $\gamma$ is the surface tension, and $k$ is the local curvature.

\section{B. Leading-order governing equation}

We require that the characteristic length in the $r$ direction, $r_{0}$ is much larger than the characteristic length in the $z$ direction, $z_{0}$,

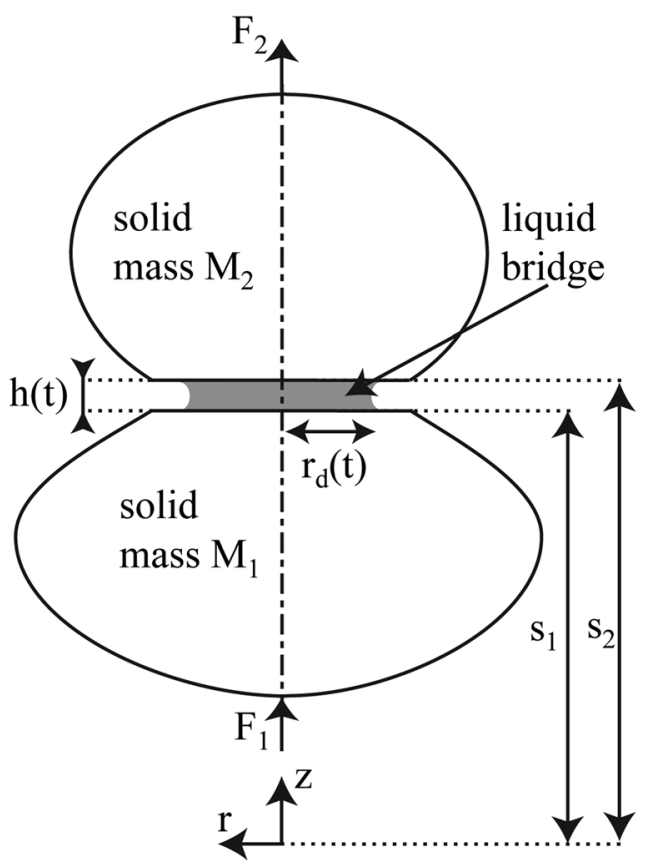

FIG. 1. A schematic description of the coordinate system and the liquid bridge (gray area) connecting two solid bodies.

$$
\varepsilon=\frac{z_{0}}{r_{0}} \ll 1
$$

Substituting the $z_{0}$ and $r_{0}$ characteristic lengths into the continuity equation and performing order of magnitude analysis we obtain

$$
\frac{v_{0}}{u_{0}} \sim \frac{z_{0}}{r_{0}}=\varepsilon,
$$

where $u_{0}$ and $v_{0}$ are the characteristic speeds in the $r$ and $z$ directions, respectively. We define a characteristic time by $t_{0} \sim r_{0} / u_{0}$. For small reduced Reynolds number, $\varepsilon \operatorname{Re}=\rho v_{0} z_{0} / \mu \ll 1$, and negligible gravity $g \rho z_{0} / p_{0} \ll 1$, order of magnitude analysis yields $u_{0}=p_{0} \varepsilon^{2} r_{0} / \mu$ where $p_{0}$ is the characteristic pressure. The value of $z_{0}$ and $r_{0}$ is defined by the gap between the surfaces at $t=0$ and the liquid volume, $V$

$$
z_{0}=\tilde{s}_{2}(t=0)-\tilde{s}_{1}(t=0), \quad r_{0}=\sqrt{\frac{\tilde{V}}{\pi z_{0}}} .
$$

Substituting these into Eq. (2) and utilizing the axisymmetry of the problem, we obtain the leading-order momentum conservation equations, ${ }^{17}$

$$
\frac{\partial p}{\partial r} \sim \frac{\partial^{2} u}{\partial z^{2}}+O\left(\varepsilon^{2},{ }_{\varepsilon} \mathrm{Re}\right)
$$

and

$$
\frac{\partial p}{\partial z} \sim O\left(\varepsilon^{2}, \varepsilon^{3} \operatorname{Re}, \frac{\rho g z_{0}}{p_{0}}\right)
$$

Assuming the viscosity of the surrounding gas is small in comparison with the liquid, $\mu^{*} \ll \mu$, the normalized dynamic stress balance at the gas-liquid interface is

$$
\mathbf{I}\left(p-p^{*}\right) \cdot \hat{\mathbf{n}}+k \frac{\varepsilon^{2}}{C a} \hat{\mathbf{n}} \sim O(\varepsilon),
$$

where $C a=\mu v_{0} / \gamma$. For the case of $C a \sim \varepsilon^{2}$, the wetting angles will approach their static advancing or receding values ${ }^{18,19}$ and for the case of $C a \gg \varepsilon^{2}$ the influence of surface tension is negligible and thus Eq. (13) is valid in the leadingorder for any value of $C a$.

As $\varepsilon \rightarrow 0$, and under the above assumptions, the shape of the liquid surface in leading-order is part of a circular arc. The curvature at the liquid-gas interface is thus obtained from geometric relations ${ }^{20}$ as $k \sim\left(\left[\cos \left(\theta_{1}\right)+\cos \left(\theta_{2}\right)\right]\right) /$ $\left(s_{2}-s_{1}\right)$, where $\theta_{1}$ and $\theta_{2}$ are the contact angles at the bottom and upper plates, respectively. Integration of Eq. (11) with regard to $z$ and the application of the no-slip boundary conditions (5) yields ${ }^{21}$

$$
u=\frac{\partial p}{\partial r}\left[\frac{\left(z-s_{1}\right)^{2}}{2}-\frac{\left(s_{2}-s_{1}\right)\left(z-s_{1}\right)}{2}\right] .
$$

We substitute $u$ into Eq. (1), integrate with regard to $z$ from $s_{1}$ to $s_{2}$, integrate with regard to $r$ and present the pressure distribution in terms of $h\left(=s_{2}-s_{1}\right)$ 


$$
\begin{aligned}
p(r, z, t)-p^{*}= & \left(r^{2}-r_{d}^{2}(t)\right) \frac{\partial h(t)}{\partial t} \frac{3}{h^{3}(t)} \\
& -\frac{\varepsilon^{2}}{C a} \frac{\cos \left(\theta_{u}\right)-\cos \left(\theta_{d}\right)}{h(t)} .
\end{aligned}
$$

Using the leading order relation $r_{d}^{2}(t) h(t) \sim r_{d}^{2}(0) h(0)$ and integrating with regard to $r$ from 0 to $r_{d}$, the force applied by the liquid on the solid bodies, $F_{l}(t)$, is related to $h(t)^{13}$

$$
F_{l}(t)=-\frac{3 \pi}{2 h^{5}(t)} \frac{\partial h(t)}{\partial t}-\pi \frac{\varepsilon^{2}}{C a} \frac{\cos \left(\theta_{u}\right)+\cos \left(\theta_{d}\right)}{h^{2}(t)}
$$

(the force acting on solids 1 and 2 is $-F_{l}$ and $F_{l}$, respectively, see Fig. 1). The magnitude of the capillary force acting at the triple phase contact line scales as $\sim \gamma r_{0}$ and is negligible in comparison with the $\sim \gamma r_{0} / \varepsilon$ force associated with the capillary pressure for the case of shallow liquid bridges. $^{20}$ Thus, the force acting in the triple phase contact line is not included in the current approximation.

For a freely moving solid body, the force applied by the liquid bridge is balanced by external and body forces acting on the solid body and the rate of change of momentum. Combining the equations of conservation of momentum for the solid bodies, we can present the momentum conservation with regard to $h$

$$
\frac{\tilde{M}_{1} \tilde{M}_{2}}{\tilde{M}_{1}+\tilde{M}_{2}} \frac{\varepsilon^{4} v_{0}}{\mu z_{0}^{2}} \frac{\partial^{2} h}{\partial t^{2}}=F_{l}(t)+\frac{\tilde{F}_{2} \tilde{M}_{1}-\tilde{F}_{1} \tilde{M}_{2}}{\tilde{M}_{1}+\tilde{M}_{2}} \frac{\varepsilon^{4}}{v_{0} z_{0} \mu} .
$$

This equation is supplemented by (but not dependent on) the conservation of momentum for the center of mass of the entire system, which is readily solved for a known $h(t)$. We thus define dimensionless mass and force by $M=\tilde{M}_{1} \tilde{M}_{2} \varepsilon^{4} v_{0} /\left(\tilde{M}_{1}+\tilde{M}_{2}\right) \mu z_{0}^{2}$ and $F=\left(\tilde{F}_{2} \tilde{M}_{1}-\tilde{F}_{1} \tilde{M}_{2}\right) \varepsilon^{4} /$ $\left(\tilde{M}_{1}+\tilde{M}_{2}\right) v_{0} z_{0} \mu$, respectively. We further simplify the equation by defining a modified capillary number,

$$
\overline{C a}=\frac{C a}{\pi \varepsilon^{2}\left[\cos \left(\theta_{u}\right)+\cos \left(\theta_{b}\right)\right]} .
$$

Equation (17) thus can be presented as

$$
M \frac{\partial^{2} h}{\partial t^{2}}+\frac{3 \pi}{2 h^{5}} \frac{\partial h}{\partial t}-F(t)+\frac{1}{\overline{C a} h^{2}}=0 .
$$

By substituting $w=\partial h / \partial t$ and assuming a constant external force, $F$, we reduce the order of the problem and Eq. (19) is transformed to the Abel equation of the second kind

$$
M w(h) \frac{\partial w(h)}{\partial h}+\frac{3 \pi}{2 h^{5}} w(h)-F+\frac{1}{\overline{C a} h^{2}}=0 .
$$

\section{Uniform asymptotic solution for the limit of small capillary numbers}

For small capillary numbers $\overline{C a} \ll 1$ and $h \sim O(1)$, viscosity is negligible in comparison with the capillary force, thus requiring (at least one of) the dimensionless mass and force values to be of order of $\sim O\left(\overline{C a}^{-1}\right)$. The governing Equation (20) is thus reduced to

$$
\overline{C a} M w(h) \frac{\partial w(h)}{\partial h}-\overline{C a} F(t)+\frac{1}{h^{2}} \sim O(\overline{C a})
$$

and the outer-region leading-order solution $w_{o}$ is, thus,

$$
w_{o}(h) \sim\left\{\left[w^{2}(h(0))\right]^{2}+2 \frac{F}{M}(h-h(0))+2 \frac{1}{\overline{C a} M}\left(\frac{1}{h}-\frac{1}{h(0)}\right)\right\}^{\frac{1}{2}} .
$$

We define an inner coordinate $H=h / \delta(\overline{C a})$ and require $H \sim O(1)$ as $\overline{C a} \rightarrow 0$. Substituting into the governing equation we obtain

$$
\frac{M w(H)}{\delta(\overline{C a})} \frac{\partial w(H)}{\partial H}+\frac{3 \pi}{2 H^{5} \delta^{5}(\overline{C a})} w(H)-F+\frac{1}{\overline{C a} \delta^{2}(\overline{C a}) H^{2}}=0 .
$$

Order of magnitude analysis yields $\delta(\overline{C a}) \sim \overline{C a}^{1 / 3}$ and thus an inner-solution is given by

$$
w_{i}(H) \sim \frac{2 H^{3}}{3 \pi} .
$$

As $h \rightarrow 0$ the outer solution $w_{O} \rightarrow \infty$ and as the stretched inner coordinate $H \rightarrow \infty$ the inner solution $w_{i} \rightarrow \infty$. Thus, in order to facilitate matching, the inner and outer solutions are multiplied by $\exp \left(-H^{2} \overline{C a}^{1 / 3}\right)$ and $\exp \left(-\overline{C a}^{1 / 3} / h^{2}\right)$, respectively. Both expressions scale as $\sim 1+O\left(\overline{\mathrm{Ca}}^{1 / 3}\right)$ in the inner and outer regions, defined by $H \sim O(1)$ and $h \sim O(1)$, respectively, and thus the multiplication does not affect the leading-order solutions in their respective region of validity. The obtained matched uniform asymptotic approximation is given by

$$
\begin{aligned}
w(h) \sim & \left\{[w(h(0))]^{2}+2 \frac{F}{M}(h-h(0))+\frac{2}{\overline{C a} M}\left(\frac{1}{h}-\frac{1}{h(0)}\right)\right\} \\
& \times \exp \left(-h^{-2} \overline{C a}^{1 / 3}\right)-\frac{2 h^{3}}{3 \pi \overline{C a}} \exp \left(-h^{2} \overline{C a}^{-1 / 3}\right) \\
& +O\left(\overline{C a}^{1 / 3}\right) .
\end{aligned}
$$

While the asymptotic solution will approach the exact solution as $\overline{C a} \rightarrow 0$, the error for a given finite value of the small parameter it is not known. We thus vary the value of $\overline{C a}$ in order to obtain a quantitative estimate of the agreement between the numerical solution of the equation and the asymptotic approximation, as well as to examine the influence of the capillary number on the $(h, \partial h / \partial t)$ curve. Fig. 2 presents the distance between the surfaces, $h$, versus the relative speed, $\partial h / \partial t$. The uniform asymptotic solution (25) and the numerical solution of the governing Equation (20) are marked by dashed and smooth lines, respectively. The physical parameters are $M=10^{2}, F=-10^{2}, w(1)=0$, and the indicated values of $\overline{C a}$. The relative differences between the asymptotic and analytic solutions scale as $\overline{\mathrm{Ca}}^{1 / 3}$ and the position of the transition between the inner and outer regions increases with the capillary number, as expected from the analysis. 

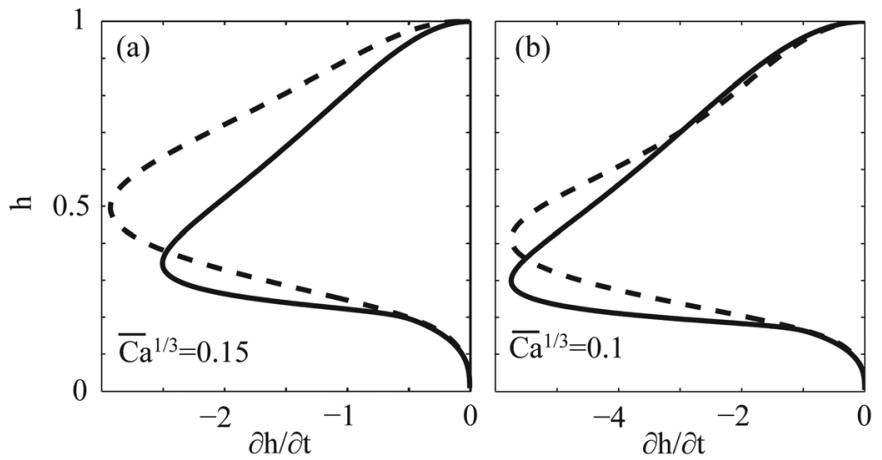

\section{Limit of large capillary numbers}

For negligible external force, $F$, and $\overline{C a} \rightarrow \infty$, the governing Equation (20) is reduced to $M \partial w(h) / \partial h \sim-3 \pi / 2 h^{5}$ and we thus obtain

$$
w-w(h(0)) \sim \frac{3 \pi}{8 M}\left(\frac{1}{h^{4}}-\frac{1}{h^{4}(0)}\right) .
$$

The limit $h(t \rightarrow \infty)$ can be presented as a function of the normalized mass, $M$, and the initial conditions $h(0)$ and $\partial h(0) / \partial t(=w(h(0)))$, by $h(t \rightarrow \infty) \sim\left(h^{-4}(0)-8 M w(0) /\right.$ $3 \pi)^{-\frac{1}{4}}$. The value of $h(t \rightarrow \infty)$ represents the distance in which the entire momentum of the solid bodies, relative to each other, is dissipated within the liquid bridge and both solid bodies move at an identical speed.

\section{E. Oscillating solutions}

For the case of different advancing and receding wetting angles, oscillations will affect the value of the capillary number. We thus define the new parameters $\overline{C a}_{M}$ and $\Delta$ by $\overline{C a}_{M}^{-1}=\left(\overline{C a}_{A}^{-1}+\overline{C a}_{R}^{-1}\right) / 2$ and $\Delta=\left(\overline{C a}_{A}^{-1}-\overline{C a}_{R}^{-1}\right) / 2$, where $\overline{C a}_{A}$ and $\overline{C a}_{R}$ are the capillary numbers based on the advancing and receding wetting angles, respectively. By introducing perturbations around the point $h_{0}=\sqrt{1 / F \overline{C a}}$, which can be described as $h \sim h_{0}+\varepsilon_{1} h_{1}$ and requiring $\varepsilon \ll \varepsilon_{1} \ll 1$, the governing equation is simplified to

$$
M \frac{\partial^{2} h}{\partial t^{2}}+\left(\frac{2 h \Delta}{h_{0}^{3}}\left|\frac{\partial h}{\partial t}\right|^{-1}+\frac{3 \pi}{2 h_{0}^{5}}\right) \frac{\partial h}{\partial t}-\frac{2 h}{h_{0}^{3} \overline{C a}_{M}} \sim 0 .
$$

Equations of the form $\partial^{2} y / \partial t^{2}+f(y) \partial y / \partial t+C y=0$ are common within the theory of nonlinear oscillations, ${ }^{22}$ suggesting oscillatory solutions in the current problem. Further-

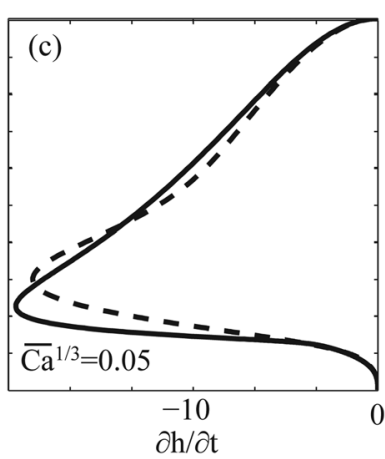

FIG. 2. Comparison between the uniform asymptotic solution (dashed lines) and numerical solution (solid lines) for the case of $w(1)=0, \quad F=-10^{2}$, $M=10^{2}$, and $\overline{\mathrm{Ca}}^{1 / 3}=0.15,0.1$ and 0.05 , corresponding to (a), (b), and (c), respectively. more, for $\Delta \rightarrow 0$, i.e., small differences between the advancing and receding wetting angles, the equation is transformed to a linear harmonic oscillator with normalized undamped angular frequency, $w_{0}$, and damping ratio, $\zeta$, estimated as

$$
w_{0} \sim \sqrt{\frac{2}{M}(-\overline{C a})^{\frac{1}{2}}(-F)^{\frac{3}{2}}}, \quad \zeta \sim \frac{3 \pi}{2} \sqrt{\frac{2}{M}(-\overline{C a})^{\frac{9}{2}}(-F)^{3}} .
$$

\section{F. Manipulation of a solid body by liquid bridges}

The manipulation of solid bodies by capillary forces commonly involves an externally controlled surface ${ }^{2-4}$ which speed and position are determined independently of the forces associated with the liquid bridge (see Fig. 3(a)). Our analysis can be readily applied to these configurations by modeling the controlled surface as a solid body with mass, $\tilde{M}_{2}$, which is order of magnitude greater than the mass of the manipulated solid body, $\tilde{M}_{1}$, and where the motion of the controlled surface is determined by selecting the value of $\tilde{F}_{2}$. (These configurations may require spacers ${ }^{1}$ or a cavity in the controlled surface ${ }^{2}$ in order to limit the minimal value of the gap between the surfaces, as illustrated in Fig. 3.)

Obata et al. ${ }^{4}$ suggested a manipulation scheme based on the capillary forces between a fixed surface, a solid body and an externally controlled surface connected by liquid bridges (see Fig. 3(b)). Using the current approximation, we can relate the speed of the controlled upper surface (defined by $\left.\partial h_{U}(t) / \partial t+\partial h_{L}(t) / \partial t\right)$ to the required movement of the manipulated solid body, $h_{L}(t)$. This yields a second-order non-linear ordinary differential equation, similar to Eq. (19)

$$
\frac{3 \pi}{2 h_{U}^{5}} \frac{\partial h_{U}}{\partial t}+\frac{1}{\overline{C a}_{U} h_{U}^{2}}=M \frac{\partial^{2} h_{L}}{\partial t^{2}}-F(t)+\frac{3 \pi}{2 h_{L}^{5}} \frac{\partial h_{L}}{\partial t}+\frac{1}{\overline{C a}_{L} h_{L}^{2}},
$$

(a)

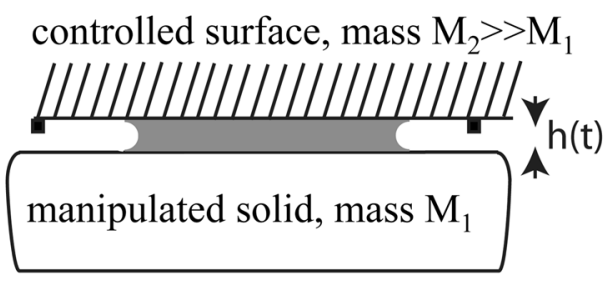

(b)

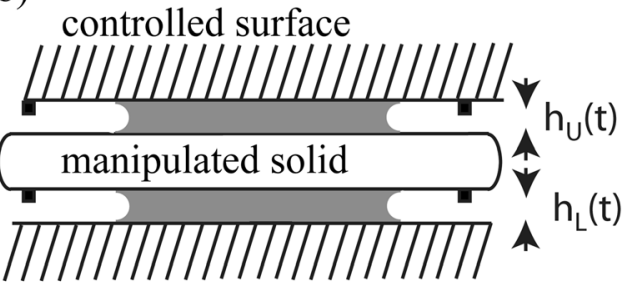

fixed surface
FIG. 3. Schematic description of a solid body connected to an externally controlled surface (a) and to both a controlled surface and a fixed surface (b). (The black squares represent spacers limiting the minimal distance between the surfaces.) 


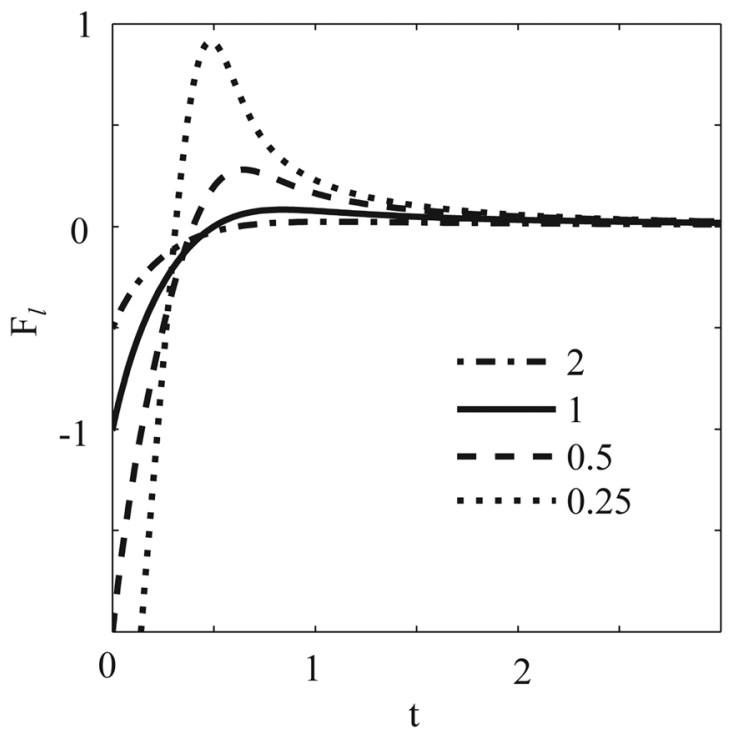

FIG. 4. $F_{l}$ vs. $\mathrm{t}$ for $\overline{C a}=0.25,0.5,1$, and 2 (dotted, dashed, solid, and dashed-dotted lines, respectively) obtained from numerical solution of Eq. (19). In all cases, $h(0)=1, \partial h(0) / \partial t=0, F=0$, and $M=1$.

where $\overline{C a}_{U}$ and $\overline{C a}_{L}$ are defined by Eq. (18) and the appropriate wetting angles of the controlled, manipulated and fixed surfaces. The parameters $M$ and $F$ are defined by $M=\tilde{M} \varepsilon^{4} v_{0} / \mu z_{0}^{2}$ and $F=\tilde{F} \varepsilon^{4} / v_{0} z_{0} \mu$, respectively, where $\tilde{M}$ is the dimensional mass of the manipulated solid and $\tilde{\mathrm{F}}$ is the dimensional force acting on the manipulated solid. For the case of small values of $\overline{C a}_{U} \ll 1$ and $h_{U} \sim O(1)$, the dissipation of momentum due to viscosity in the upper liquid bridge is negligible and the governing equation is simplified, thus yielding an explicit approximation for $h_{U}(t)$

$$
h_{U} \sim \sqrt{\frac{1}{\overline{C a}_{U}}}\left(M \frac{\partial^{2} h_{L}}{\partial t^{2}}-F(t)+\frac{3 \pi}{2 h_{L}^{5}} \frac{\partial h_{L}}{\partial t}+\frac{1}{\overline{C a}_{L} h_{L}^{2}}\right)^{-\frac{1}{2}} .
$$

\section{RESULTS}

\section{A. Oscillations and impulse-like spikes in the force}

Fig. 4 presents the force acting on the plates as a function of time for $F=0, M=1, \overline{C a}=0.25,0.5$, 1 , and 2 (dotted, dashed, smooth, and dashed-dotted lines, respectively) obtained from numerical solution of Eq. (19). In all cases, $h(0)=1$ and $\partial h(0) / \partial t=0$. For greater values of the capillary number $(\overline{C a}=2)$, the applied force is attractive throughout the movement of the solid bodies. However, as the capillary number $\overline{C a}$ decreases a positive peak in the force, created by the viscous resistance of the liquid, is evident and increases as $\overline{C a}$ decreases. This behavior may appear to be counterintuitive, since the value of the capillary number increases with viscous resistance. It is, however, explained within the context of the uniform asymptotic approximation developed in Sec. II C, describing an inner and outer regions for the limit of small $\overline{C a}$. Viscous resistance is a negligible in the outer region and a dominant mechanism in the inner region. The inner region is defined by $h \sim \overline{C a}^{1 / 3}$ and thus as the capillary number decreases the viscous resistance is concentrated at a smaller region, creating impulse-like spikes of viscous repulsive force between the plates for the limit of $\overline{C a}$.

Fig. 5 focuses on small capillary numbers and presents $h$ versus time $(a, b)$ and force vs. time $(c, d)$. The physical parameters are $\overline{C a}=10^{-2}(\mathrm{a}, \mathrm{c}),-10^{-2}(\mathrm{~b}, \mathrm{~d})$, the mass is $M=10^{2}, 10^{3}$, and $10^{4}$ (dotted, dashed, and smooth, respectively) and the external force is $F=-10^{3}$. We observe impulse-like spike in the force applied by the liquid bridge in the transition between the inner and outer-regions. The magnitude of the impulse increases with $M$, although the external force and capillary attraction remain unchanged. For the negative values of $\overline{C a}$ (wetting angles of $\theta>90^{\circ}$ ), oscillations of the system occur and the distance between the plates asymptotes to a finite value which is independent of the value of $M$.

\section{B. Comparison with experiments}

The experimental setup consists of a linear stage actuator (Thorlabs $^{\mathrm{TM}}$ LNR50SEK1) controlling the distance between two parallel plates, a load cell (Interface ${ }^{\mathrm{TM}} \mathrm{MB}-$ LBF5) measuring the force acting on the solid body and two optical windows (Newport ${ }^{\mathrm{TM}}$ 20BW40-30) used as the upper and lower plates (see Fig. 6). A feedback control loop was created, connecting the load-cell force input, the actuator position and speed inputs and outputting the upper plate acceleration and speed. The feedback loop was programed to simulate a freely moving solid body with a given mass $\tilde{M}_{1}$, reacting to external forces and the force applied by a liquid bridge connected to a solid body with mass $\tilde{M}_{2} \rightarrow \infty$. The plates are cleaned before each experiment by Acetone, Isopropanol, and Nitrogen gas. The advancing and receding wetting angles were estimated from quasi-static force measurements (method suggested and validated by de Souza et $\left.a l .{ }^{14}\right)$. Fig. 7 presents experimental and theoretical values of the force applied by static liquid bridge, for both advancing and receding contact angles, on the supporting solid plates (de Souza et al. ${ }^{14}$ ). Square, circle, and triangle signs mark experimental data for the case of advancing water, advancing mercury, and receding mercury contact angles, respectively. The experiments utilized $10^{-7}\left[\mathrm{~m}^{3}\right]$ DI-water droplet and a $5 \cdot 10^{-8}\left[\mathrm{~m}^{3}\right]$ mercury droplet. The wetting angle was estimated from least square minimization of the discrepancies between the analytic model and the experimental measurements, yielding contact angle values of $75^{\circ}, 120^{\circ}$, and $116^{\circ}$ for advancing water (smooth line), advancing mercury (dashed line), and receding mercury (dotted line), respectively.

Fig. 8 presents experimental results for $3 \cdot 10^{-8}\left[\mathrm{~m}^{3}\right]$ DI water liquid bridge with advancing wetting angle of $75^{\circ}$ with regard to the fused-silica plates and physical properties of $\mu=10^{-3}\left[\mathrm{pa}^{1} \mathrm{~s}^{1}\right], \gamma=0.072\left[\mathrm{~J}^{1} \mathrm{~m}^{-2}\right]$, and $\rho=10^{3}\left[\mathrm{Kg}^{1} \mathrm{~m}^{-3}\right]$. The plates are positioned at $\tilde{h}(0)=5 \cdot 10^{-4}[\mathrm{~m}]$ with initial relative speed $\partial \tilde{h}(0) / \partial \tilde{t}=0$ and the movement of the plates was limited to $\tilde{h}(0)>5 \cdot 10^{-5}[\mathrm{~m}]$. The feedback control loop was programmed to simulate solid bodies with mass of $\tilde{M}_{1}=500[K g]$ and $\tilde{M}_{2} \rightarrow \infty$ (connected to the ground) 

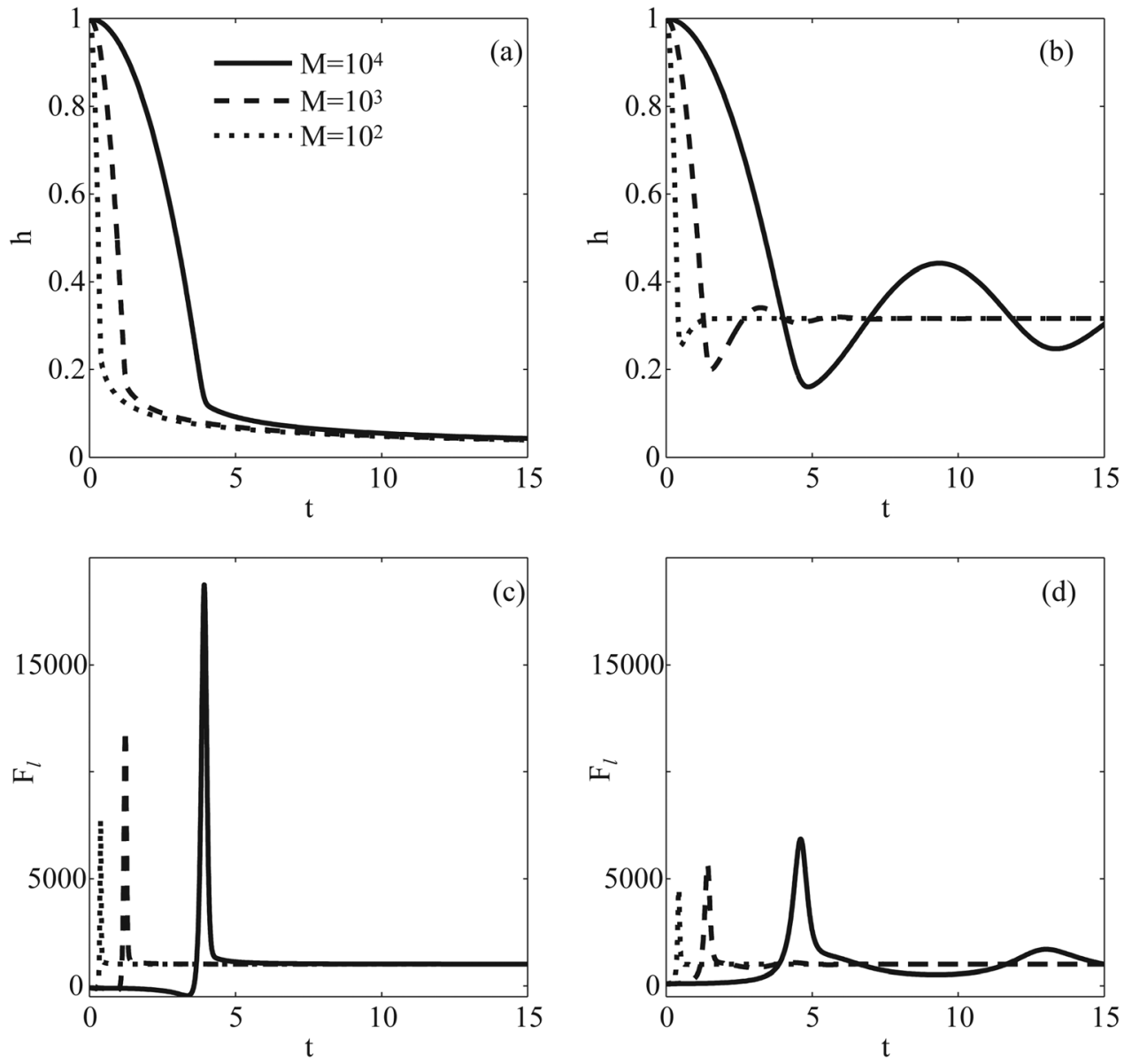

FIG. 5. The gap $h$ between the solid bodies $(\mathrm{a}, \mathrm{b})$ and the force $F_{l}$ applied by the liquid on the solid bodies (c,d) vs. $t$ for $\overline{C a}=10^{-2}(\mathrm{a}, \mathrm{c})$ and $\overline{C a}=-10^{-2}$ $(b, d)$. In all cases, the normalized physical parameters are $h(0)=1, \partial h(0) / \partial t=0$, $F=-10^{3}, M=-10^{2}, 10^{3}$, and $10^{4}$ (dotted, dashed, and solid lines, respectively). without any external forces or gravity. Fig. 9 presents the experimental results for the case of liquid mercury with the physical parameters $\tilde{h}(0)=1.5 \cdot 10^{-3}[m], \quad \partial \tilde{h}(0) / \partial \tilde{t}=0$, $\tilde{M}_{1}=100[\mathrm{~kg}], \quad \tilde{M}_{2} \rightarrow \infty, \quad \tilde{F}_{1}=-0.2[N], \quad \tilde{V}=10^{-7}\left[\mathrm{~m}^{3}\right]$, $\mu=1.5 \cdot 10^{-3}\left[p a^{1} s^{1}\right], \quad \gamma=0.486\left[J^{1} m^{-2}\right], \quad$ and $\quad \rho=$ $1.35 \cdot 10^{4}\left[\mathrm{~kg}^{1} \mathrm{~m}^{-3}\right]$. These parameters were chosen in order to slow the time scale of the experiment and thus allow for higher resolution of the experimental results (see Eq. (28)).

A reasonable agreement between the analytic model (smooth line, Eq. (20)) and the experimental data (dashed line) is evident in both Figs. 8 and 9, where the experimental uncertainty is marked by the gray lines. In Fig. 8(a), we (a)

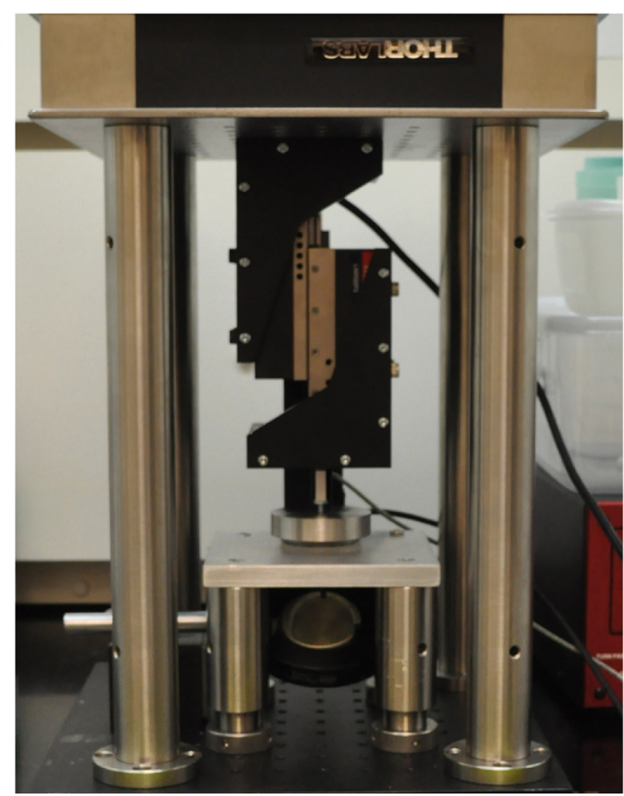

(b)

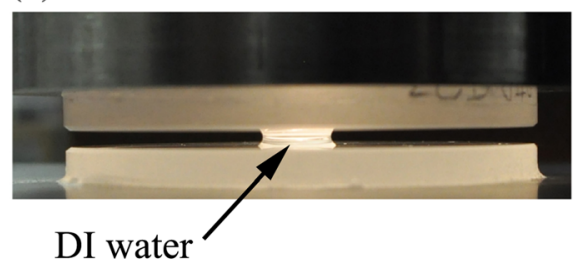

(c)

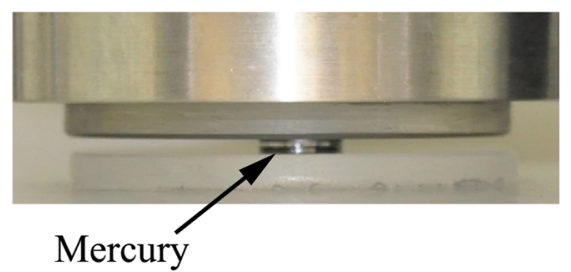

FIG. 6. (Color online) The experimental setup (a), a DI water liquid bridge (b), and mercury liquid bridge (c) connecting two fused-silica plates. 


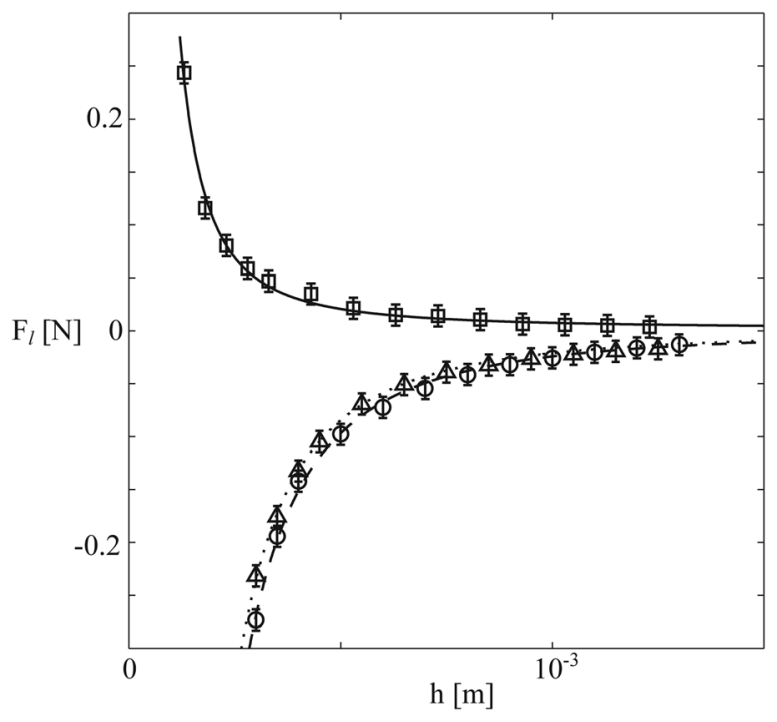

FIG. 7. Estimation of advancing and receding contact angles from quasistatic force measurement of liquid bridges (following de Souza et al. ${ }^{14}$ ). Square, circle, and triangle signs mark experimental force measurements of advancing water, advancing mercury, and receding mercury contact angles. The experiments utilized $10^{-7}\left[\mathrm{~m}^{3}\right]$ DI-water droplet and a $5 \cdot 10^{-8}\left[\mathrm{~m}^{3}\right]$ mercury droplet. The smooth, dashed, and dotted lines mark theoretical data for force applied by a static liquid bridge (de Souza et al. ${ }^{14}$ ), where the wetting angle was obtain from least squares optimization with regard to the relevant experimental results.

observe a sharp transition from capillary attraction between the plates to the spike-like impulse in the repulsive force due to viscous resistance, as expected from the analysis. Fig. 8(b) presents the relative speed between the plates, $\partial h / \partial t$, as a function of the separation gap $h$ in order to enable comparison with the uniform asymptotic approximation (Eq. (25); Fig. 2). As mentioned in Sec. II C, for $h \rightarrow 0$ the outer solution $w_{o} \rightarrow \infty$ and as the stretched inner coordinate $H \rightarrow \infty$ the inner solution $w_{i} \rightarrow \infty$. This in turn creates a maxima point of the speed between the plates, representing the transition between the inner and outer regions. The characteristic speed and length scale are $z_{0} \sim 5 \cdot 10^{-4}[\mathrm{~m}]$ and $u_{0} \sim 10^{-4}[\mathrm{~m} / \mathrm{s}]$, respectively, yielding a capillary number of the order of $\overline{C a} \sim 10^{-4}$. The predicted maxima in the relative speed between the plates are observed at $h \sim \overline{C a}^{1 / 3}$ in Fig. 8(b), similarly to the results presented in Fig. 2.
In Fig. 9, we observe oscillations with regard to $h$ (part a), the gap between the plates, and the associated spikes of the force applied by the liquid bridge (part b). Comparing the frequency and damping ratio of the experimental oscillations to the values estimated by the linear harmonic oscillator approximation, Eq. (28) yields similar values of the oscillation frequency (theoretical angular frequency of $\mathrm{w}_{0}=2.85[\mathrm{rad} / \mathrm{s}]$ and experimental value of $\left.\mathrm{w}_{0} \approx 2.4[\mathrm{rad} / \mathrm{s}]\right)$. However, the damping ratio is different by order of magnitude relative to the value given by Eq. (28), suggesting that the main mechanism for damping emanates from the non-linear term associated with the difference between the advancing and receding wetting angles. We also observe small oscillations persisting after the decay of the initial oscillations, unpredicted by the model, and with different oscillation frequency compared with the initial oscillations. From visual observations of the liquid mercury triple phase contact line, it is evident that the contact line is fixed and the oscillations are of the wetting angle only. Since these oscillations are of first-order, they are not explained by the current leading-order model and more research is required on this subject.

\section{CONCLUDING REMARKS}

For small capillary numbers there is an impulse-like peak in the force applied on the flat surfaces by the liquid bridge, occurring in the transition between the inner- and outer-region. Oscillations may occur for the case of low wettability of the liquid (wetting angle $>90^{\circ}$ ) and the frequency of the oscillations has been estimated by the linearization of the governing equation. The damping of the oscillations is related to the viscous resistance and to the nonlinear term associated with the difference between the advancing and receding wetting angles. Using the current approximation, a second-order non-linear ordinary differential equation describing the motion of a solid body connected by liquid bridges to two flat planes is obtained and can be used to facilitate analysis for the manipulation of solid bodies by capillary forces. ${ }^{4}$

The results of the current analysis suggest several possible future research directions, including: (1) Analysis of a
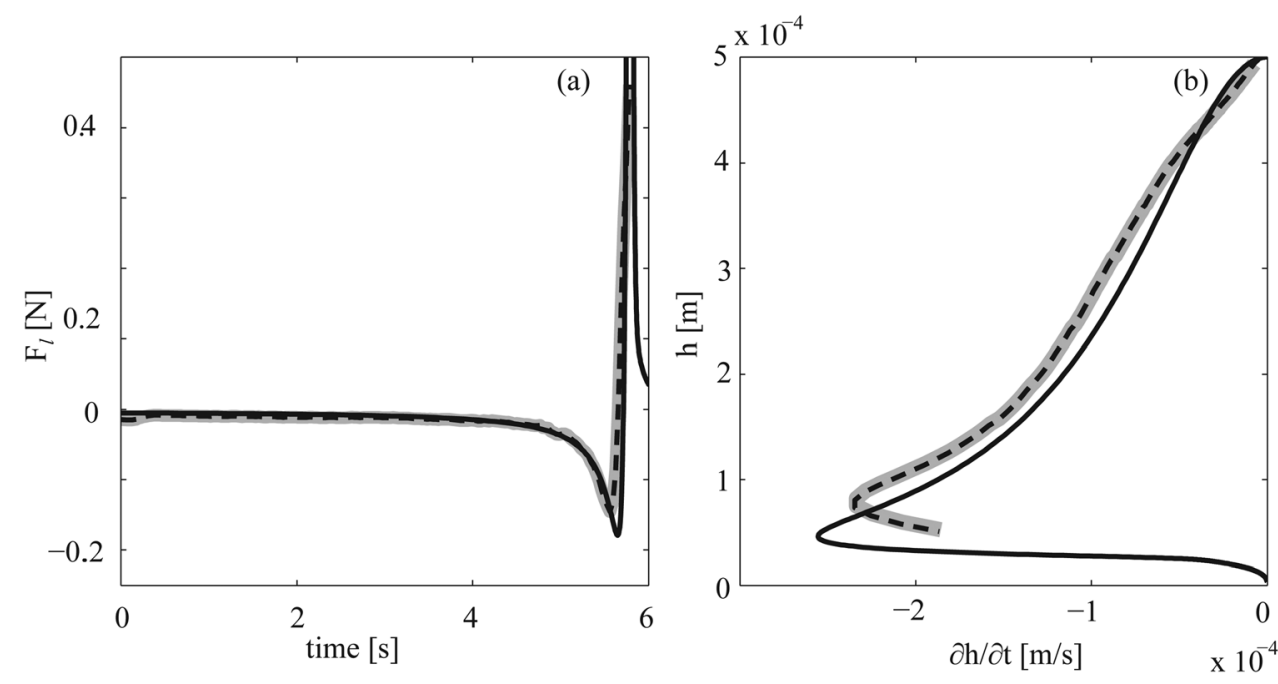

FIG. 8. Comparison of $F_{l}$ vs. $t$ (a) and $h$ vs. $\partial h / \partial t$ (b) between theoretical (solid lines, Eq. (20)) and experimental (dashed lines) results. The gray lines mark the uncertainty of the experimental measurements. The physical parameters of the experiment are $\tilde{h}=5 \cdot 10^{-4}[\mathrm{~m}], \partial \tilde{h}(0) /$ $\partial \tilde{t}=0, \quad \tilde{M}_{1}=500[\mathrm{~kg}], \quad \tilde{M}_{2} \gg \tilde{M}_{1}$, $\tilde{V}=3 \cdot 10^{-8}\left[\mathrm{~m}^{3}\right], \quad \mu=10^{-3}\left[\mathrm{pa}^{1} \mathrm{~s}^{1}\right]$, $\gamma=0.072\left[\mathrm{~J}^{1} \mathrm{~s}^{-2}\right]$, and $\rho=10^{3}\left[\mathrm{~kg}^{1} \mathrm{~m}^{-3}\right]$. The advancing wetting angle is $75^{\circ}$ (calculated from static force measurements) and the experiment was stopped at $\tilde{h}=5 \cdot 10^{-5}[\mathrm{~m}]$. 

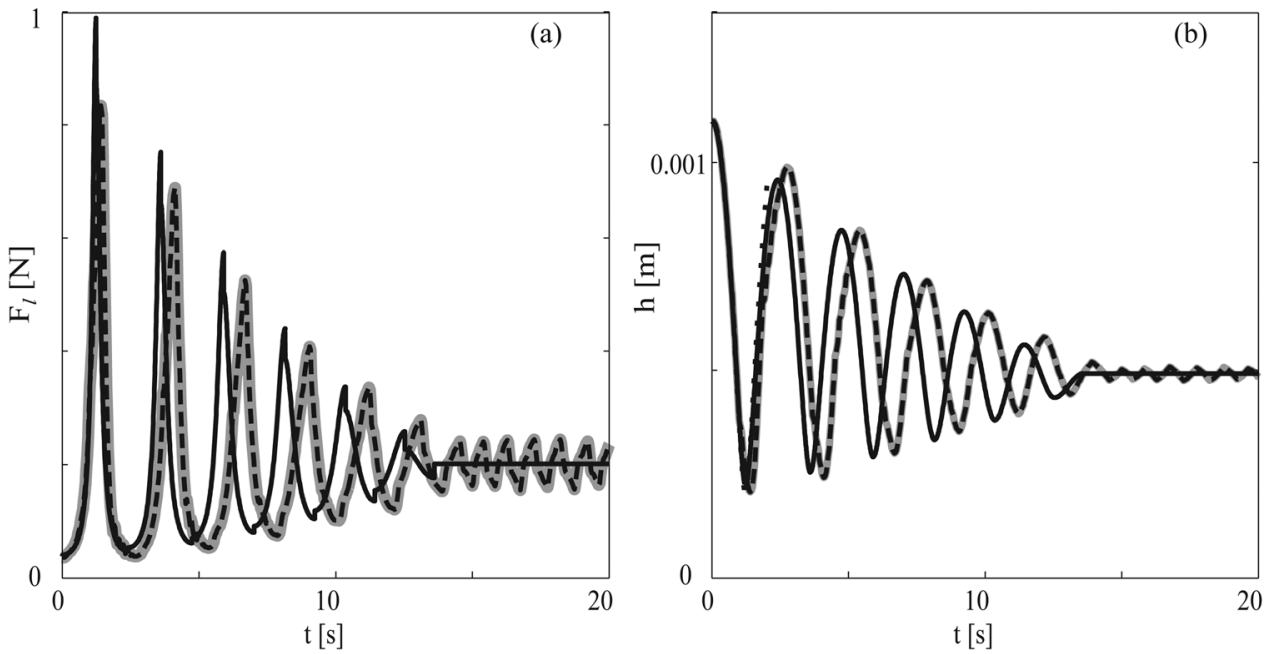

FIG. 9. Comparison of $F_{l}$ vs. $t$ (a) and $h$ vs. $t$ (b) between the theoretical model (solid lines), and experimental data (dashed lines). The gray lines mark the uncertainty of the experimental measurements. The physical parameters of the experiment are $\tilde{h}(0)=1.5 \cdot 10^{-3}[\mathrm{~m}]$, $\partial \tilde{h}(0) / \partial \tilde{t}=0, \quad \tilde{M}_{1}=100[\mathrm{Kg}], \quad \tilde{M}_{2}$ $\gg \tilde{M}_{1}, \quad \tilde{F}_{1}=-0.2[N], \quad \tilde{V}=10^{-7}\left[\mathrm{~m}^{3}\right]$, $\mu=1.5 \cdot 10^{-3}\left[\mathrm{pa}^{1} \mathrm{~s}^{1}\right], \gamma=0.486\left[\mathrm{~J}^{1} \mathrm{~m}^{-2}\right]$, and $\quad \rho=1.35 \cdot 10^{4}\left[\mathrm{~kg}^{1} \mathrm{~m}^{-3}\right]$. The advancing and receding wetting angles are $\theta_{a}=120^{\circ}$ and $\theta_{r}=116^{\circ}$ (calculated from static force measurements). liquid bridge as a controlled shock absorber, where the damping will be modified by changing the wetting angles (e.g., by electric fields). (2) Use of the oscillations and the associated force and pressure spikes to enhance diffusion into porous materials (drug delivery). This can be achieved by creating perturbations in the natural frequency of the system. (3) Obtaining solutions for the equation describing the manipulation of a solid body and extending the analysis to non-flat surfaces. (4) Analysis of the first-order oscillations of the wetting angle observed in the experimental data.

\section{ACKNOWLEDGMENTS}

We thank Dr. Sari Paikoff and Dr. James Savage of the Defense Threat Reduction Agency (DTRA) which supported the project. The authors wish to acknowledge the advice of Adrianus Aria regarding the development of the experimental setup.

${ }^{1}$ M. J. Vogel and P. H. Steen, "Capillarity-based switchable adhesion," Proc. Natl. Acad. Sci. U.S.A. 107, 3377 (2010).

${ }^{2}$ S. Saito, T. Motokado, K. J. Obata, and K. Takahashi, "Capillary force with a concave probe-tip for micromanipulation," Appl. Phys. Lett. 87, 234103 (2005).

${ }^{3}$ P. Lambert, F. Seigneur, S. Koelemeijer, and J. Jacot, "A case study of surface tension gripping: The watch bearing," J. Micromech. Microeng. 16, 1267 (2006).

${ }^{4}$ K. J. Obata, T. Motokado, S. Saito, and K. Takahashi, "A scheme for micro-manipulation based on capillary force," J. Fluid Mech. 498, 113 (2004).

${ }^{5} \mathrm{G}$. Fantoni and M. Porta, "A Critical review of releasing strategies in microparts handling,” Int. Fed. Inf. Process.: Micro-Assem. Technol Appl. 260, 223 (2008).
${ }^{6}$ P. Lambert, M. Mastrangeli, J.-B. Valsamis, and G. Degrez, "Spectral analysis and experimental study of lateral capillary dynamics for flip-chip applications," Microfluid. Nanofluid. 9, 797 (2010).

${ }^{7}$ B. Markicevic, T. G. D'Onofrio, and H. K. Navaz, "On spread extent of sessile droplet into porous medium: Numerical solution and comparisons with experiments," Phys. Fluids 22, 012103 (2010).

${ }^{8}$ H.-J. Butt and M. Kappl, "Normal capillary forces," Adv. Colloid Interface Sci. 146, 48 (2008).

${ }^{9}$ W. C. Carter, "The forces and behavior of fluids constrained by solids," Acta Metall. 36, 2283 (1988).

${ }^{10}$ D. N. Mazzon, G. I. Tardos, and R. Pfeffe, "The behavior of liquid bridges between two relatively moving particles," Powder Technol. 51, 71 (1987).

${ }^{11}$ S. Dodds, M. D. S. Carvalho, and S. Kumar, "Stretching and slipping of liquid bridges near plates and cavities," Phys. Fluids, 21, 092103 (2009).

${ }^{12}$ O. Pitois, P. Moucheront, and X. Chateau, "Liquid bridge between two moving spheres: An experimental study of viscosity effects," J. Colloid Interface Sci. 231, 26 (2000).

${ }^{13}$ M.-H. Meurisse and M. Querry, "Squeeze effects in a flat liquid bridge between parallel solid surfaces," J. Tribol. 128, 575 (2006).

${ }^{14}$ E. J. De Souza, L. Gao, T. J. McCarthy, E. Arzt, and A. J. Crosby, "Effect of contact angle hysteresis on the measurement of capillary forces," Langmuir 24, 1391 (2008).

${ }^{15}$ W. M. Deen, Analysis of Transport Phenomena (Oxford University Press, New York, 1998).

${ }^{16}$ L. G. Leal, Advanced Transport Phenomena (Cambridge University Press, New York, 2007).

${ }^{17}$ A. Oron, S. H. Davis, and S. G. Bankoff, "Long-scale evolution of thin liquid films," Rev. Mod. Phys. 69, 931 (1997).

${ }^{18}$ P.-G. de Gennes, F. Brochard-Wyart, and D. Quere, Capillarity and Wetting Phenomena (Springer, New York, 2004).

${ }^{19}$ S. Sikalo, C. Tropea, and E. Ganic, "Dynamic wetting angle of a spreading droplet," Exp. Therm. Fluid Sci. 29, 795 (2005).

${ }^{20}$ E. J. De Souza, M. Brinkmann, C. Mohrdieck, A. Crosby, and E. Arzt, "Capillary forces between chemically different substrates," Langmuir 24, 10161 (2008).

${ }^{21}$ H. Schlichting, Boundary Layer Theory (McGraw-Hill, New York, 1968).

${ }^{22}$ A. Fidlin, Nonlinear Oscillations in Mechanical Engineering (Springer, New York, 2006). 